\title{
A theoretical approach for the interpretation of pulsating PMS intermediate-mass stars
}

\author{
A. Ruoppo ${ }^{1,2}$, M. Marconi ${ }^{1}$, J. P. Marques ${ }^{3,4,5}$, M. J. P. F. G. Monteiro ${ }^{4,5}$, J. Christensen-Dalsgaard ${ }^{6}$, \\ F. Palla ${ }^{7}$, and V. Ripepi ${ }^{1}$ \\ 1 INAF - Osservatorio Astronomico di Capodimonte, via Moiariello 16, 80131 Napoli, Italy \\ e-mail: [ruoppo;marconi;ripepi]@na.astro.it \\ 2 Dipartimento di Scienze Fisiche, Università Federico II, Complesso Monte S. Angelo, 80126 Napoli, Italy \\ ${ }^{3}$ Grupo de Astrofísica da Universidade de Coimbra, Departamento de Matemática - FCTUC, Portugal \\ 4 Departamento de Matemática Aplicada, Faculdade de Ciências da Universidade do Porto, Portugal \\ 5 Centro de Astrofísica da Universidade do Porto, Rua das Estrelas, 4150-762 Porto, Portugal \\ e-mail: [jmarques;mjm]@astro.up.pt \\ ${ }^{6}$ DASC and Institut for Fysik og Astronomi, Aarhus Universitet, Denmark \\ e-mail: jcd@phys .au.dk \\ 7 INAF - Osservatorio Astrofisico di Arcetri, Largo E. Fermi 5, 50125 Firenze, Italy \\ e-mail: palla@arcetri.astro.it
}

Received 16 November 2006 / Accepted 29 January 2007

\section{ABSTRACT}

\begin{abstract}
Context. The investigation of the pulsation properties of pre-main-sequence intermediate-mass stars is a promising tool to evaluate the intrinsic properties of these stars and to constrain current evolutionary models. Many new candidates of this class have been discovered during the last decade and very accurate data are expected from space observations obtained for example with the CoRoT satellite.

Aims. In this context we aim at developing a theoretical approach for the interpretation of observed frequencies, both from the already available ground-based observations and from the future more accurate and extensive CoRoT results.

Methods. To this purpose we have started a project devoted to the computations of fine and extensive grids of asteroseismic models of intermediate mass pre-main-sequence stars. The obtained frequencies are used to derive an analytical relation between the large frequency separation and the stellar luminosity and effective temperature and to develop a tool to compare theory and observations in the echelle diagram.

Results. The predictive capabilities of the proposed method are verified through the application to two test stars. As a second step, we apply the procedure to two true observations from multisite campaigns and we are able to constrain their stellar parameters, in particular the mass, in spite of the small number of frequencies.

Conclusions. We expect that with a significantly higher number of frequencies both the stellar mass and age could be constrained and, at the same time, the physics of the models could be tested.
\end{abstract}

Key words. stars: variables: $\delta$ Sct - stars: oscillations - stars: pre-main sequence - stars: fundamental parameters

\section{Introduction}

Pre-main-sequence (PMS) $\delta$ Scuti are intermediate-mass (1.5 $M_{\odot}<M<4 M_{\odot}$ ) stars that cross the pulsation instability strip of more evolved classical pulsators during their evolution toward the main sequence (MS). The first evidence of $\delta$ Scuti pulsation in PMS stars was provided by Breger (1972), who discovered two candidates in the young cluster NGC 2264. More than 20 years elapsed since this discovery before other authors confirmed the occurrence of this kind of pulsation observing the Herbig Ae stars HR5999 (Kurtz \& Marang 1995) and HD104237 (Donati et al. 1997). In the same years Marconi \& Palla (1998) obtained the first theoretical instability strip for PMS intermediate-mass pulsators by computing an extensive and detailed set of nonlinear convective models for the first three radial modes, along the PMS evolutionary tracks by Palla \& Stahler (1993). More recently Grigahcéne et al. (2006) have produced a theoretical instabily strip for PMS stars for the first seven radial modes. Marconi \& Palla (1998) also pointed out that the interior structure of PMS stars entering the instability strip differs significantly from that of more evolved main-sequence stars (with the same mass and temperature), even though the structure of the envelope is similar. This property was subsequently confirmed by Suran et al. (2001) who made a comparative study of the seismology of a $1.8 M_{\odot}$ PMS and post-MS star and found that the unstable frequency range is roughly the same for PMS and post-MS stars, but that some non-radial modes are very sensitive to the deep internal structure of the star. In particular, it is possible to discriminate between the PMS and post-MS stage using differences in the oscillation frequency distribution in the low frequency range ( $g$ modes; see also Templeton \& Basu 2003).

The theoretical analysis by Marconi \& Palla (1998) stimulated an increasing observational interest for this class of pulsators and the current number of known or suspected candidates amounts to about 30 stars. An updated list is available at http://ams.astro.univie.ac.at/pms_corot.php, and there are recent reviews by Zwintz et al. (2004), Marconi \& Palla (2004) and Ripepi et al. (2006b). However, only a few stars have 
been studied in detail, so that the overall properties of this class of variables are still poorly determined. In this context our group has started a systematic monitoring program of Herbig Ae stars with spectral types from A to F2-3 with the following aims: 1) to identify the largest number of pulsating objects in order to observationally determine the boundaries of the instability strip for PMS $\delta$ Scuti pulsation; 2) to study in detail through multisite campaigns selected objects showing multiperiodicity (see Marconi et al. 2001; Ripepi et al. 2002, 2003, 2006a; Pinheiro et al. 2003; Bernabei et al. 2004) which are the best potential candidates for asteroseismology.

In order to establish a theoretical approach for the interpretation of these data, as well as to prepare the basis for the interpretation of future more accurate satellite observations expected from CoRoT (Baglin \& the CoRoT Team 2003), in the context of the CoRoT/ESTA collaboration (http://www.astro.up.pt/corot/) we have started a theoretical project devoted to the computation of asteroseismic models of intermediate mass PMS stars covering a wide range of stellar masses. In this paper we present our first results based on an extensive grid of evolutionary PMS models computed with the CESAM code (Morel 1997), with updated physics (Marques et al. 2004) relevant for PMS modelling. The Aarhus linear adiabatic non-radial pulsation code ADIPLS (http://astro.phys.au.dk/ jcd/adipack.n/) has been applied to the models of the grid in order to build a reference base to reproduce the observed pulsation frequencies.

The paper is organized as follows: in Sect. 2 we present the evolutionary PMS models, we introduce the adopted non-radial pulsation code and report on the predicted pulsation frequencies; in Sect. 3 the computed large separation and its dependence on luminosity and effective temperature are discussed; in Sect. 4 we present a method to reproduce the observed frequencies and a theoretical test of the predictive capabilities of the method; in Sect. 5 we compare the theoretical results with the observations from multisite campaigns of the stars V351 Ori and IP Per. Finally the Conclusions close the paper.

\section{Reference grid of models with pulsation frequencies}

For exploring the pulsation properties of intermediate-mass PMS stars, we decided to build an extensive grid of models and frequencies to provide a reference on the seismic characteristics of PMS stars across the HR diagram.

\subsection{PMS evolution models}

The stellar models have been computed with the CESAM code (Morel 1997) that is optimized for asteroseismology. The precise calculation of oscillation frequencies ( $p$ and $g$ modes) requires numerically precise and adequately dense meshes with the quantities describing the internal structure and entering the oscillation equations (e.g Christensen-Dalsgaard \& Berthomieu 1991). The physical and numerical properties of these models are discussed in detail in the paper by Morel (1997) with an updated version of the physics being used as in Marques et al. (2004). From the CESAM PMS evolutionary tracks (see Fig. 3) we selected 56 models for stellar masses varying from 1.6 to $4.0 M_{\odot}$ with a step of $0.2 M_{\odot}$. For each selected mass, we consider from two to four different effective temperatures in the range 6000 to $10000 \mathrm{~K}$, along the corresponding evolutionary track. We report
Table 1. Physical properties of the selected PMS models.

\begin{tabular}{|c|c|c|c|c|c|}
\hline Model & $\begin{array}{c}\text { Mass } \\
M / M_{\odot}\end{array}$ & $\begin{array}{l}\text { Radius } \\
R / R_{\odot}\end{array}$ & $\begin{array}{c}\text { Luminosity } \\
L / L_{\odot}\end{array}$ & $\begin{array}{r}T_{\text {eff }} \\
\mathrm{K} \\
\end{array}$ & $\begin{array}{r}\text { Age } \\
\text { Gy }\end{array}$ \\
\hline $\bmod 1$ & 1.6 & 1.78 & 7.18 & 7078 & 11.21 \\
\hline $\bmod 2$ & 1.6 & 1.52 & 6.72 & 7548 & 16.82 \\
\hline $\bmod 3$ & 1.8 & 2.32 & 15.23 & 7493 & 7.86 \\
\hline $\bmod 4$ & 1.8 & 1.78 & 9.70 & 7648 & 9.83 \\
\hline $\bmod 5$ & 1.8 & 2.13 & 14.83 & 7773 & 8.30 \\
\hline $\bmod 6$ & 1.8 & 1.61 & 11.59 & 8401 & 11.80 \\
\hline $\bmod 7$ & 2.0 & 2.75 & 21.93 & 7539 & 5.93 \\
\hline $\bmod 8$ & 2.0 & 1.88 & 14.27 & 8184 & 7.41 \\
\hline $\bmod 9$ & 2.0 & 1.70 & 18.40 & 9186 & 8.89 \\
\hline $\bmod 10$ & 2.2 & 3.67 & 18.70 & 6273 & 4.07 \\
\hline $\bmod 11$ & 2.2 & 3.10 & 30.04 & 7681 & 4.66 \\
\hline $\bmod 12$ & 2.2 & 1.99 & 20.74 & 8749 & 5.82 \\
\hline $\bmod 13$ & 2.2 & 1.77 & 27.27 & 9913 & 6.98 \\
\hline $\bmod 14$ & 2.4 & 4.14 & 24.06 & 6288 & 3.25 \\
\hline $\bmod 15$ & 2.4 & 3.48 & 39.04 & 7739 & 3.72 \\
\hline $\bmod 16$ & 2.4 & 2.09 & 29.46 & 9304 & 4.65 \\
\hline $\bmod 17$ & 2.4 & 2.04 & 37.54 & 10000 & 5.11 \\
\hline $\bmod 18$ & 2.6 & 4.55 & 33.38 & 6506 & 2.82 \\
\hline $\bmod 19$ & 2.6 & 3.92 & 48.15 & 7688 & 3.10 \\
\hline $\bmod 20$ & 2.6 & 3.54 & 57.56 & 8459 & 3.24 \\
\hline $\bmod 21$ & 2.6 & 3.14 & 66.90 & 9319 & 3.38 \\
\hline $\bmod 22$ & 2.6 & 2.18 & 43.08 & 10023 & 4.09 \\
\hline $\bmod 23$ & 2.8 & 4.81 & 46.39 & 6876 & 2.40 \\
\hline $\bmod 24$ & 2.8 & 4.46 & 55.53 & 7468 & 2.51 \\
\hline $\bmod 25$ & 2.8 & 4.08 & 66.50 & 8168 & 2.63 \\
\hline $\bmod 26$ & 2.8 & 3.23 & 90.99 & 9885 & 2.85 \\
\hline $\bmod 27$ & 3.0 & 5.58 & 46.31 & 6381 & 1.90 \\
\hline $\bmod 28$ & 3.0 & 4.96 & 64.92 & 7366 & 2.08 \\
\hline $\bmod 29$ & 3.0 & 4.17 & 91.89 & 8758 & 2.26 \\
\hline $\bmod 30$ & 3.0 & 3.74 & 107.9 & 9621 & 2.35 \\
\hline $\bmod 31$ & 3.0 & 3.32 & 122.2 & 10547 & 2.45 \\
\hline $\bmod 32$ & 3.2 & 6.13 & 53.10 & 6301 & 1.57 \\
\hline $\bmod 33$ & 3.2 & 5.49 & 74.04 & 7230 & 1.73 \\
\hline $\bmod 34$ & 3.2 & 4.67 & 105.7 & 8573 & 1.88 \\
\hline $\bmod 35$ & 3.2 & 4.22 & 125.5 & 9417 & 1.96 \\
\hline $\bmod 36$ & 3.2 & 3.75 & 146.2 & 10375 & 2.04 \\
\hline $\bmod 37$ & 3.4 & 6.46 & 69.66 & 6567 & 1.37 \\
\hline $\bmod 38$ & 3.4 & 5.76 & 94.50 & 7502 & 1.49 \\
\hline $\bmod 39$ & 3.4 & 4.96 & 130.4 & 8762 & 1.61 \\
\hline $\bmod 40$ & 3.4 & 4.53 & 152.5 & 9539 & 1.67 \\
\hline $\bmod 41$ & 3.4 & 4.08 & 176.0 & 10414 & 1.73 \\
\hline $\bmod 42$ & 3.6 & 7.29 & 67.80 & 6139 & 1.09 \\
\hline $\bmod 43$ & 3.6 & 6.46 & 100.3 & 7194 & 1.24 \\
\hline $\bmod 44$ & 3.6 & 5.71 & 134.7 & 8234 & 1.33 \\
\hline $\bmod 45$ & 3.6 & 4.88 & 180.9 & 9590 & 1.43 \\
\hline $\bmod 46$ & 3.6 & 4.44 & 207.6 & 10402 & 1.48 \\
\hline $\bmod 47$ & 3.8 & 7.84 & 81.15 & 6194 & 0.94 \\
\hline $\bmod 48$ & 3.8 & 6.94 & 116.3 & 7203 & 1.06 \\
\hline $\bmod 49$ & 3.8 & 6.21 & 152.9 & 8155 & 1.14 \\
\hline $\bmod 50$ & 3.8 & 5.40 & 202.2 & 9376 & 1.22 \\
\hline $\bmod 51$ & 3.8 & 4.97 & 231.2 & 10106 & 1.26 \\
\hline $\bmod 52$ & 4.0 & 8.25 & 101.0 & 6375 & 0.83 \\
\hline $\bmod 53$ & 4.0 & 7.30 & 140.7 & 7369 & 0.92 \\
\hline $\bmod 54$ & 4.0 & 6.58 & 180.5 & 8253 & 0.98 \\
\hline $\bmod 55$ & 4.0 & 5.80 & 232.9 & 9366 & 1.05 \\
\hline $\bmod 56$ & 4.0 & 5.40 & 263.8 & 10023 & 1.08 \\
\hline
\end{tabular}

in Table 1 the physical properties of the selected PMS models, which cover the empirical HR diagram location of known pulsating PMS intermediate-mass stars.

\subsection{Calculation of the oscillation frequencies}

In order to explore the pulsation frequencies that characterize the selected PMS models, associated to both radial and non-radial 


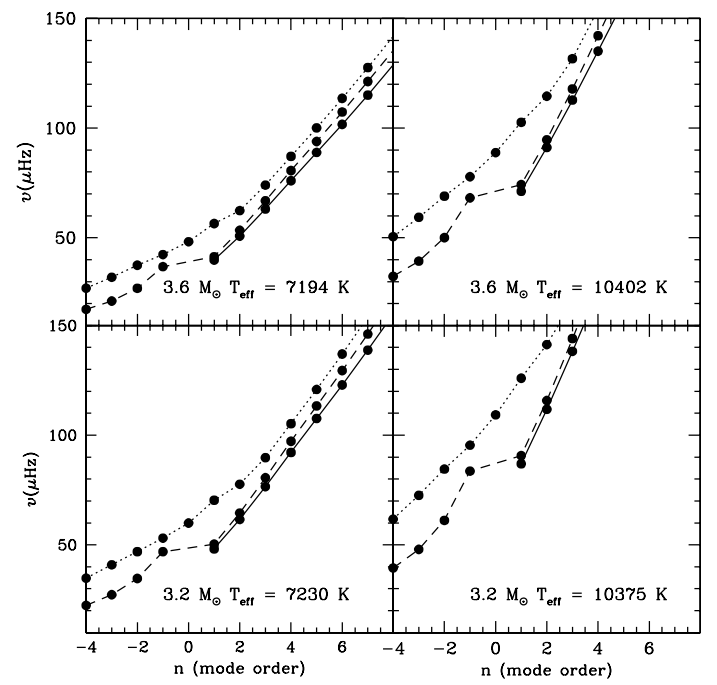

Fig. 1. Predicted frequencies as a function of the radial order $n$ for models at $3.2 M_{\odot}$ (bottom panel: $\bmod 33$ left, $\bmod 36$ right), and $3.6 M_{\odot}($ upper panel: mod43 left, mod46 right). Symbols are connected by a full line in the case of $l=0$, by a dashed line for $l=1$ and by a dotted line for $l=2$. The effective temperatures of the models are indicated for each mass.

modes, we applied the ADIPLS code. The pulsation frequencies are obtained as solutions of a set of linear equations for small perturbations (for details see Unno et al. 1979).

The physical and numerical assumptions used for the computation are discussed in detail by Christensen-Dalsgaard (1982); Christensen-Dalsgaard \& Berthomieu (1991); ChristensenDalsgaard \& Mullan (1994).

In this work we concentrate mainly on $p$ modes, i.e. acoustic modes, with a spherical harmonic degree $l=0,1,2$ and radial order $n=0,1, \ldots, 20$. We only consider very low degree modes because these are thought to be more likely detectable (e.g. Dziembowski 1977; Christensen-Dalsgaard \& Gough 1982). From a first inspection of the results we note that the predicted range of $p$-mode frequencies moves toward higher values as the stellar mass decreases, at fixed effective temperature, and as the effective temperature increases, at fixed stellar mass (see Fig. 1). This behaviour confirms the well known relation between the pulsation frequency and the stellar mean density.

\section{The large frequency separation}

The pattern of regular separations between frequencies of modes of different degree and order is a characteristic feature expected in $p$-mode stellar oscillations. The frequency separations depend on the internal structure of the star so by measuring them we gain valuable diagnostics on the stellar interior.

In particular, in the asymptotic regime, it is convenient to use the large frequency separation defined as

$\Delta v_{n, l} \equiv v_{n, l}-v_{n-1, l} \simeq \Delta v$,

between modes of the same order, where $\Delta v \simeq\left(\int_{0}^{R} \mathrm{~d} r / c\right)^{-1} \propto$ $\left(M / R^{3}\right)^{1 / 2}$, with $c$ the sound speed in the star.

In Fig. 2 we show for model mod 8 the behaviour of the large separation as defined in Eq. (1), for $l=0,1,2$, versus the frequency.

On this basis a relation can be found between the large frequency separation, the luminosity and the effective temperature (the stellar mass is related to the luminosity for each effective

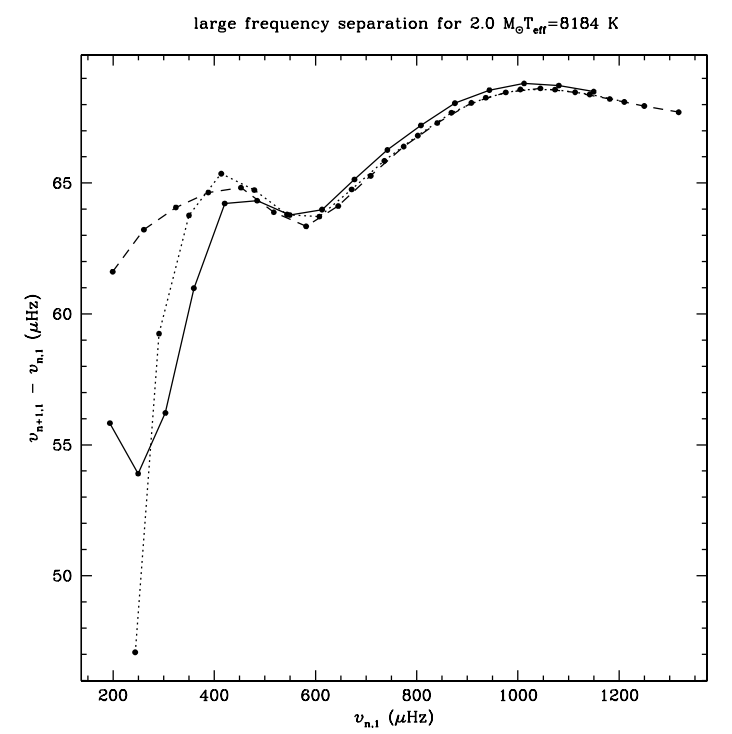

Fig. 2. Large frequency separation as a function of frequency for the model with $M=2.0 M_{\odot}$ and $T_{\text {eff }}=8184 \mathrm{~K}$, for $1<n \leq 18$. Symbols are connected by a full line in the case of $l=0$, by a dashed line for $l=1$ and by a dotted line for $l=2$.



Fig. 3. HR diagram showing PMS evolutionary tracks computed with the CESAM code for the labelled stellar masses (in $M_{\odot}$ ) The linear fit given in Eq. (2) is represented by dashed lines, defined for a constant value of $\Delta v$ (as labelled, in $\mu \mathrm{Hz}$ ), from $20 \mu \mathrm{Hz}$ to $70 \mu \mathrm{Hz}$.

temperature). For this purpose, we have computed a large separation $\Delta v$ in the asymptotic region as the mean of all values for $5<n \leq 20$.

A linear fit for all models in our grid gives the following relation:

$$
\begin{aligned}
\log L= & (-12.81 \pm 0.02)+(4.35 \pm 0.05) \log T_{\text {eff }} \\
& -(1.681 \pm 0.015) \log \Delta v
\end{aligned}
$$

plotted in the HR diagram in Fig. 3, for selected values of $\Delta v$, ranging from $20 \mu \mathrm{Hz}$ to $70 \mu \mathrm{Hz}$. This relation is useful to obtain an expected range of the large separation of an observed pulsating star once its position in the HR diagram has been estimated. In the next section we use this approach as a first step to characterise a PMS $\delta$ Scuti pulsator with the adopted non-radial pulsation models and the observed frequencies. 
Table 2. Physical properties (exact values) of the two test stars. These values were only known to one member of the team. The quantities provided to the other team members are listed as "Observed" values.

\begin{tabular}{lccccc}
\hline \hline & $\begin{array}{c}\text { Mass } \\
M / M_{\odot}\end{array}$ & $\begin{array}{c}\text { Radius } \\
R / R_{\odot}\end{array}$ & $\begin{array}{c}\text { Luminosity } \\
\log \left(L / L_{\odot}\right)\end{array}$ & $\begin{array}{c}\log \left(T_{\text {eff }}\right) \\
\mathrm{K}\end{array}$ & $\begin{array}{c}\text { Age } \\
\text { My }\end{array}$ \\
\hline \multicolumn{2}{l}{ Exact values: } & & & & \\
Star1 & 2.0 & 1.862 & 1.1942 & 3.9251 & 0.00 \\
Star2 & 2.4 & 3.482 & 1.5915 & 3.8887 & 3.72 \\
\hline "Observed" values: & & & & \\
Star1 & & $1.19 \pm 0.82$ & $3.92 \pm 0.06$ & \\
Star2 & & $1.58 \pm 0.07$ & $3.88 \pm 0.05$ & \\
\hline
\end{tabular}

\section{How to reproduce the observed frequencies}

In this section we present a methodology to compare the observed pulsation frequencies with theoretical ones. This comparison allows us to obtain information on the stellar structure and physical parameters, such as the mass, the radius, the effective temperature and the age. For simulating typical available data for observed stars, we "theoretically built" two PMS test stars. The first one, Starl, corresponds to a model computed using the STAROX code (Roxburgh 2005) as determined for Task 1 of CoRoT/ESTA, while the second one, Star2, was obtained with the CESAM code (Morel 1997) after integration of the birthline from Palla \& Stahler (1993) as the initial configuration for the evolution calculated by CESAM (Marques 2006). The parameters characterising these models are given in Table 2.

We adopted two different evolutionary codes for producing the two test objects (also changing the initial condition in one of these) because we need to ensure that the use of the same code for building the test cases and the grid of models used here to estimate the stellar parameters is not the dominant factor determining the success of our inversion procedure. The physical properties of these two test stars are presented in Table 2, whereas the corresponding theoretical frequencies, for both radial and nonradial $p$ and $g$ pulsation modes, were computed using the POSC code (Monteiro 1996) - again a different code from the one used to calculate the frequencies of the reference grid. The values of the frequencies, the spherical harmonic degree $l$ and the radial order $n$, are reported in Table 3 .

We note that most of these frequencies have been verified to correspond to modes that are actually unstable, through the use of nonadiabatic computations. We also notice that only one author knew the real stellar parameters and the mode parameters of the frequencies, while the others tried to find the best solution reproducing the observed stellar parameters and frequencies, just as if they were true observed data. In particular, the author that knew the properties of the two test stars has simulated real data by shifting the frequencies, the luminosity and the effective temperature relatively to the exact value and quoting an error not always compatible with the original (exact) value. The frequencies for $g$ modes have also been added in one case. All values, exact and simulated observations, are reported in Tables 2-3.

In both test cases the number of frequencies is high (10 or more) and with a few of those already in the asymptotic regime. Present data on PMS $\delta$ Scuti stars are not so rich (only a few frequencies) but forthcoming observations from CoRoT are expected to provided several tens of frequencies per star. To illustrate the present situation we consider later an application to two real cases observed in multisite campaigns.
Table 3. Frequencies, spherical harmonic degree, and the radial order as computed using the POSC code for the two test stars. In the last column the "observed" frequencies provided as observables for the two test stars are also given.

\begin{tabular}{rcccc}
\hline \hline & $\begin{array}{c}v_{n, l} \\
\mu \mathrm{Hz}\end{array}$ & $l$ & $n$ & "Observed" $f_{i}$ \\
$\mu \mathrm{Hz}$
\end{tabular}

Based on the "observed" data we have tried to establish if we could determine the basic stellar parameters. In doing so an approach has been developed in order to best use the available data when there is no mode identification and poorly constrained stellar parameters (as is often the case for single PMS stars). To summarize our approach the steps we follow are:

- combine the grid of models with the known luminosity and effective temperature to determine a range in mass and expected large frequency separation;

- estimate the large frequency separation from the frequency data;

- reduce the mass range by using the observed large frequency separation;

- use a few models within the possible range of mass and large separation to reproduce the frequencies in the echelle diagram;

- based on the best representation in the echelle diagram able to reproduce the observations we provide the preferred stellar parameters and a tentative mode identification.

This sequence is motivated by the need to use sequentially the most robust information contained in the frequencies. This is done by reducing as much as possible the effects on the analysis of the unknown physics that is not included in the reference grid of models and frequencies.

The proposed sequence of steps is executed for our two test stars as described in the next sections. 


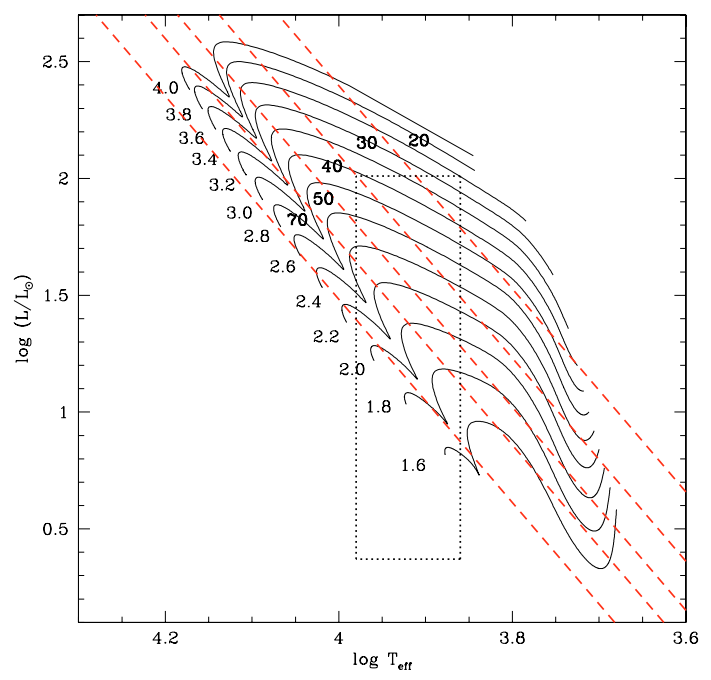

Fig. 4. HR diagram position of Starl (dotted square) together with CESAM PMS evolutionary tracks (full lines, labelled with masses in $M_{\odot}$ ) and the linear fit (dashed lines, labelled with $\Delta v$ in $\mu \mathrm{Hz}$ ) of constant large frequency separation obtained in Sect. 3.

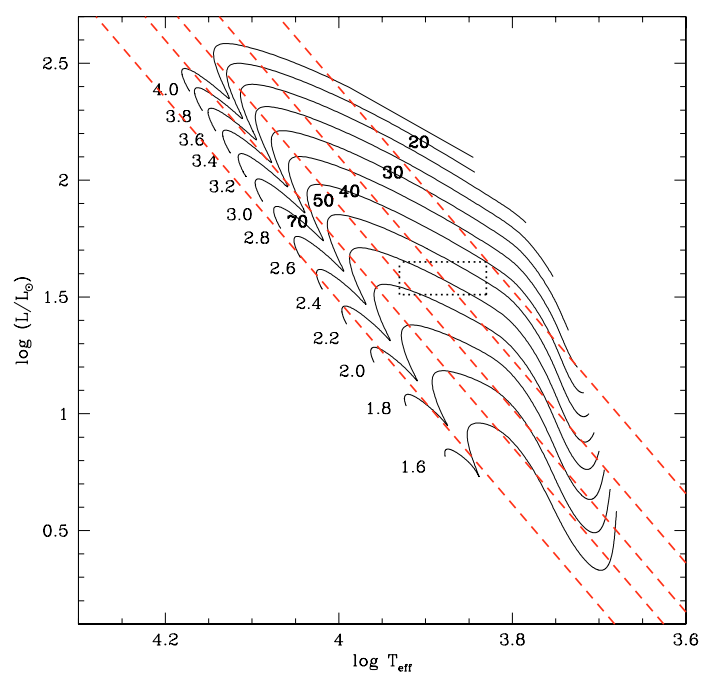

Fig. 5. The same as Fig. 4, but for Star2.

\subsection{The HR diagram}

On the basis of the reported range of luminosity and effective temperature we show in the Figs 4-5 the predicted HR diagram position of Starl and Star2 respectively. In the same plots we also display the set of PMS evolutionary tracks from CESAM for stellar masses from 1.6 to 4.0 solar masses, together with the linear fit obtained in the previous section for constant large separations (ranging from 20 to $70 \mu \mathrm{Hz}$ ). These plots allow us to define the range in mass consistent with each star and determine the possible range of values for the large separation. The obtained mass and large separation ranges are:

Starl : $1.6 \leq M / M_{\odot} \leq 3.4$ and $20 \mu \mathrm{Hz} \leq \Delta v \leq 80 \mu \mathrm{Hz}$,

Star2 : $2.2 \leq M / M_{\odot} \leq 2.8$ and $20 \mu \mathrm{Hz} \leq \Delta v \leq 50 \mu \mathrm{Hz}$.

These are yet large ranges of possible values which correspond to the uncertainty on stellar mass when only the classical observables (luminosity and effective temperature) are available.

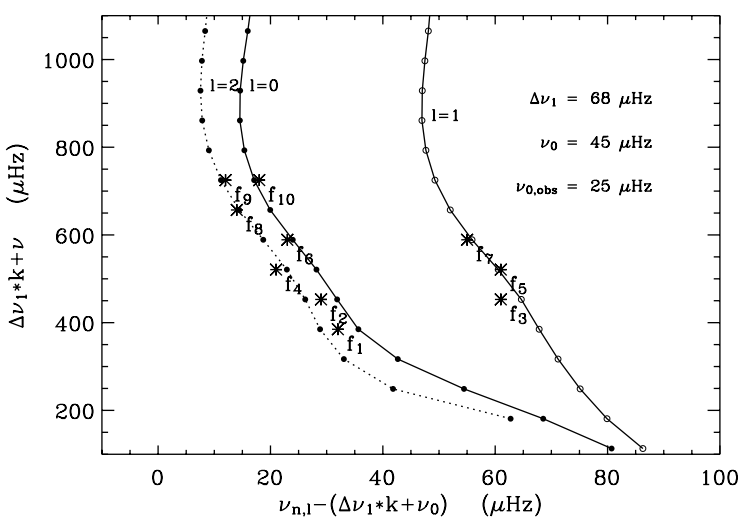

Fig. 6. Echelle diagram for Starl, using the theoretical frequencies computed with the Aarhus code. The parameters used to produce this plot are shown in the plot. The observational uncertainties would correspond approximately to the size of the symbols representing the observed frequencies $f_{i}$.

\subsection{The large frequency separation}

In a real case the large separation would be ideally extracted directly from the power spectrum of the light curve based on the expected range reported above. The development of an extraction method for this quantity from the power spectrum, based on the methodology developed for solar-type stars but adapted to $\delta$ Scuti PMS stars, will be described elsewhere.

For the test cases we have here, there is not a power spectrum so an alternative analysis has to be used in order to estimate the large separation from the actual frequencies. Based on the regularity of the spacing between frequencies we obtain an indication of the large separations as being about $\Delta v_{1} \sim 65 \mu \mathrm{Hz}$ and $\Delta v_{2} \sim 27 \mu \mathrm{Hz}$ for Starl and Star2, respectively. This approach does not provide an uncertainty but in a real case this 'error' could be as high as $\sigma(\Delta v) \sim 5 \mu \mathrm{Hz}$.

\subsection{The echelle diagram}

As it is well known, the echelle diagram is convenient to illustrate in detail the properties of the frequency spectrum. In this diagram the frequencies are reduced modulo $\Delta v$ by expressing them as

$v_{n, l}=v_{0}+k \Delta v+\tilde{v}$

where $\Delta v$ is the large separation, $v_{0}$ is a suitably chosen reference, and $k$ is an integer such that $\tilde{v}$ is between 0 and $\Delta v$. The diagram is produced by plotting $\tilde{v}$ on the abscissa and $v_{0}+k \Delta v$ on the ordinate. If the asymptotic relation between frequencies and large separation were precisely satisfied, we would obtain data points arranged on a set of vertical lines corresponding to the different values of $l$.

By using the best values for the large separation, determined before, we select all the models for which the intersection of the corresponding constant $\Delta v$ lines with the evolutionary tracks are inside the assumed uncertainty box (see Figs. 4-5). For the models located at these intersections we use the computed frequencies to construct theoretical echelle diagrams and search for the one that best reproduces (i.e. matches as many as possible of) the observed frequencies. As a result we obtain $M=2.0 M_{\odot}$, $T_{\text {eff }}=8184 \mathrm{~K}$ for Starl and $M=2.4 M_{\odot}, T_{\text {eff }}=7739 \mathrm{~K}$ for Star2. The corresponding echelle diagrams are shown in Figs. 6-7. 


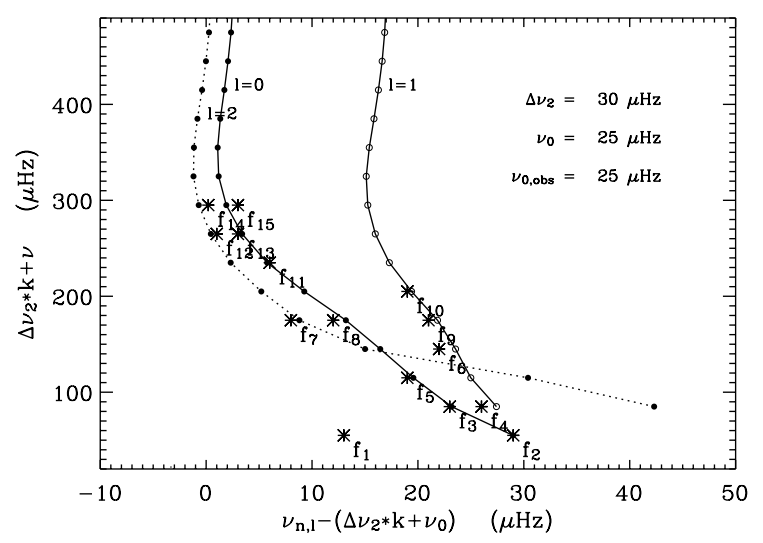

Fig. 7. The same as Fig. 6, but for Star2.

\subsection{Results}

As shown in Fig. 6, for Starl all the frequencies are reproduced with our best fit model, and we are able to obtain the correct spherical harmonic degree $l$ and radial order $n$, for each frequency. Our best fit model has also the right mass and an effective temperature that differs from the true one by only $200 \mathrm{~K}$. We remind that this is the case with a poorly determined luminosity, but whose impact on the mass estimation is weak. Based on the mode identification provided by this approach, a more detailed study of the global characteristics and the internal structure of the star could now be attempted.

Also for Star 2 we are able to reproduce the right mass and effective temperature and all the 16 frequencies with the right $l$ and $n$ degrees (see Fig. 7). The only exception is $f_{1}$ that corresponds to a $g$ mode and this is the reason why the echelle diagram has been unable - correctly - to adjust this particular value, even if our best-fitting model has a $g$ mode that fits $f_{1}$ within $0.005 \mu \mathrm{Hz}$.

These are both ideal test cases, but they demonstrate that if enough frequencies are known with sufficiently high precision, the oscillation spectra can be interpreted using as reference a detailed grid of PMS models and their frequencies as proposed in this work. The key assumption is that the measured values of the frequencies do not deviate strongly from the predicted values and relative spacing. Any effect that changes significantly the linear adiabatic radial and non-radial frequencies used here as the reference may invalidate this assumption. These can include fast rotation and strong magnetic effects on the frequencies. Before a definite characterisation of the observed modes is reached, these effects need to be taken into account on a caseby-case basis.

The heavy-element abundance $Z$ (or metallicity) is also an important parameter of the grid (here $Z=0.02$ has been used) that has an impact on the outcome of the fitting. This parameter can, however, be included in the proposed procedure either after the last step by re-building the echelle diagram with a model with an adequate $Z$ or by using a reference grid of models calculated with representative metallicities.

\section{Preliminary comparison with observations}

Having tested our theoretical approach on artificial pulsators, we may now try to evaluate how it can be applied to real observations. The difficulty with ground-based observations of PMS $\delta$ Scuti stars is that these do not provide yet a large set of frequencies for each star. But space observations have already been
Table 4. Observed frequencies and associated uncertainties for V351 Ori, as reported by Ripepi et al. (2003).

\begin{tabular}{ccr}
\hline \hline & $\begin{array}{c}\text { Frequency } \\
\mu \mathrm{Hz}\end{array}$ & $\begin{array}{c}\text { Error } \\
\mu \mathrm{Hz}\end{array}$ \\
\hline$f_{1}$ & 181.6 & 0.4 \\
$f_{2}$ & 165.9 & 0.4 \\
$f_{3}$ & 147.6 & 0.4 \\
$f_{4}$ & 183.8 & 0.4 \\
$f_{5}$ & 148.3 & 0.4 \\
\hline
\end{tabular}

reported where several tens of frequencies with very high precision (Matthews 2007) are measured for a single star. These will be the ideal cases for applying the approach described above.

\subsection{The case of V351 Ori}

As a preliminary application we considered the known multiperiodic PMS $\delta$ Scuti star V351 Ori (see Ripepi et al. 2003). The observed frequencies for this object, with the associated uncertainties, are reported in Table 4. As noted by Ripepi et al. (2003) the first four frequencies are well established, whereas $f_{5}$ is slightly less reliable. Moreover $f_{4}$ and $f_{5}$ are quite close to $f_{1}$ and $f_{3}$ respectively. As discussed by Breger \& Bischof (2002) close frequency pairs should be investigated accurately in order to establish whether they are real or not. In particular the quoted authors suggest a method to discriminate which hypothesis (close pair or single mode with amplitude variation) is correct. Unfortunately, current data on V351 Ori are insufficient to follow this approach. Consequently we will mainly focus on reproducing frequencies $f_{1}, f_{2}$ and $f_{3}$.

The ranges of luminosity and effective temperature as reported in the literature for V351 Ori are plotted in the HR diagram (dotted box) in Fig. 8 with the PMS evolution tracks. Similarly to the plots presented for the two test stars, we also report in the same diagram the lines at constant large frequency separation. This comparison allows us to obtain a range in mass and large separation consistent with the estimated HR diagram position of the star, namely $1.8<M / M_{\odot}<3.0$ and $20 \mu \mathrm{Hz}<$ $\Delta v_{a}<50 \mu \mathrm{Hz}$.

The next step is to identify from the frequency data if a stronger constraint can be posed on the large frequency separation. The large separation of V351 Ori is difficult to estimate directly from the power spectrum. Instead, we use the separations between observed frequencies to evaluate for what conditions the modes with larger amplitude (expected to be unstable loworder radial modes) are consistent with the range of $\Delta v$ found from the HR diagram. By using the reference grid we can extrapolate to the asymptotic regime to find that the frequencies are consistent with a $\Delta v_{a} \sim 40 \mu \mathrm{Hz}$.

Consequently, to find the best model that reproduces the observed frequencies we built the echelle diagram by varying the mass and the large separation within the constraints indicated above. In doing so we assume that the mode with the largest amplitude $\left(f_{1}\right)$ is a radial mode. As a result, we find that the model in the grid consistent with the observed frequencies has a mass $M=2.0 M_{\odot}$ and an effective temperature of $T_{\text {eff }}=7539 \mathrm{~K}$. As shown in the echelle diagram (see Fig. 9), the frequencies $f_{1}$ and $f_{3}$ are associated with radial modes $(l=0)$, namely to the first and second overtone respectively, while $f_{2}$ is a nonradial mode (rather close to the $l=2$ theoretical sequence). As for $f_{4}$ and $f_{5}$ their position in the echelle diagram is between the $l=0$ and the $l=1$ sequences, but as discussed above and in particular for $f_{5}$, 




Fig. 8. The same as Fig. 4, but for stars V351 Ori (box with dotted square line), IP Per (box with dot-dashed line).

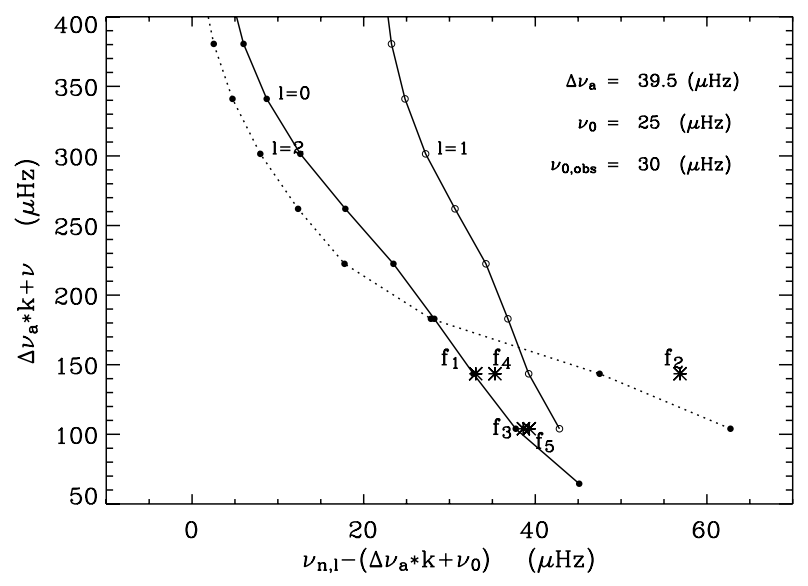

Fig. 9. The same as Fig. 6, for V351 Ori.

the true nature should be investigated carefully on the basis of more extensive data.

There are key assumptions that were made that require further verification. One is the assumption that the highest amplitude mode $\left(f_{1}\right)$ is a radial mode. This is required to properly adjust the echelle diagram to the observed spectrum. This hypothesis, or an equivalent calibration for a radial mode, requires verification from spectroscopic mode identification.

Rotation also introduces an additional difficulty as it may change the mode identification we obtain for non-radial modes and shift the frequencies. In particular, for our best-fitting model the rotational splitting should be of the order of $8 \mathrm{~m} / \sin i \mu \mathrm{Hz}$ for an estimated rotation velocity $v \sin i \simeq 100 \mathrm{~km} \mathrm{~s}^{-1}$ (see Balona et al. 2002). Such a relatively large splitting removes the possibility that the separation between $f_{4}$ and $f_{1}$ and between $f_{3}$ and $f_{5}$ is due to rotation, once the hypothesis of radial modes for $f_{1}$ and $f_{3}$ is abandoned. However, $f_{2}$ is shifted by about $10 \mu \mathrm{Hz}$ from the predicted frequency, which may correspond to a $m \neq 0$ mode, pending spectroscopic confirmation. It should also be noted that for such a relatively rapid rotation higher-order effects must be taken into account (e.g., Gough \& Thompson 1990; Dziembowski \& Goode 1992; Soufi et al. 1998). These
Table 5. Observed frequencies and associated uncertainties for IP Per, as reported by Ripepi et al. (2006a).

\begin{tabular}{ccr}
\hline \hline & $\begin{array}{c}\text { Frequency } \\
\mu \mathrm{Hz}\end{array}$ & $\begin{array}{r}\text { Error } \\
\mu \mathrm{Hz}\end{array}$ \\
\hline$f_{1}$ & 264.9 & 1.3 \\
$f_{2}$ & 400.5 & 1.3 \\
$f_{3}$ & 352.4 & 1.3 \\
$f_{4}$ & 558.2 & 1.3 \\
$f_{5}$ & 333.2 & 1.3 \\
$f_{6}$ & 277.6 & 1.3 \\
$f_{7}$ & 107.6 & 1.3 \\
$f_{8}$ & 487.4 & 1.3 \\
$f_{9}$ & 602.3 & 1.3 \\
\hline
\end{tabular}



Fig. 10. The same as Fig. 6, for IP Per.

lead to non-uniform splitting and shifts of modes with $m=0$, including the radial modes. It is likely that such rotational frequency perturbations will be a substantial complication in the analysis of oscillations of PMS stars, which typically are rapid rotators.

\subsection{The case of IP Per}

Another case that we may also consider as a possible preliminary application is IP Per, which has been the target of a recent multisite campaign (Ripepi et al. 2006a). The observed frequencies are listed in Table 5 while in Fig. 8 the HR diagram includes the location of the star (box with dot-dashed line) and the reference grid of evolution tracks. From this plot we can restrict the mass and large separation expected for this star to the ranges $1.6<M / M_{\odot}<2$ and $50 \mu \mathrm{Hz}<\Delta v_{b}<75 \mu \mathrm{Hz}$.

Due to the higher number of modes, and considering that five of these have a large amplitude, the separation between observed frequencies indicates that the data are consistent, within the expected range, with $\Delta v_{b} \sim 50 \mu \mathrm{Hz}$, when extrapolated to the asymptotic regime. Following these constraints we have then used the echelle diagram to find the best stellar mass and age that reproduce as many as possible of the observed frequencies. As a result we find a best fit model with $M=1.8 M_{\odot}$, and $T_{\text {eff }}=7773 \mathrm{~K}$. The corresponding echelle diagram is shown in Fig. 10.

According to this plot, frequencies from $f_{1}$ to $f_{4}$ seem to align along the sequence for $l=0$ modes, whereas $f_{5}, f_{6}$ and $f_{8}$ are better in agreement with $l=1$ theoretical predictions, and $f_{9}$ could be an $l=2$ mode. The discrepancy between the theoretical and observed frequencies is probably due to a residual 
uncertainty on stellar mass, intrinsic to the adopted model grid. A slightly lower mass would probably allow us to better reproduce the observed frequencies in the echelle diagram. In any case the frequency $f_{7}$ is not consistent with a $p$ mode as it lies well below the expected frequencies for the range of stellar masses of IP Per. This frequency could correspond to a $g$ mode, but we have not explored this possibility further here.

\section{Conclusions}

In this work we establish a procedure based on an extended grid of models and oscillation frequencies to analyse the properties of PMS $\delta$ Scuti stars. The procedure uses a grid of PMS evolution models to identify in the HR diagram the range of stellar mass. From the frequencies we estimate the large frequency separation which is then used to reduce the uncertainty on stellar mass. Finally, from the detailed analysis of the echelle diagram for the few possible combinations of mass/age, a fit of as many observed frequencies as possible is obtained. This fit provides an initial guess on the mode parameters and a precise estimation of the stellar mass. The underlying principle of this approach is to extract sequentially the information from the frequencies that are more robust and directly connected to the global parameters, reducing in this way as much as possible the effect on the inferences of the unknown physics in this type of stars. We also notice that the proposed method is quite general, as it can be applied by using models computed with any evolutionary code.

In order to evaluate the validity of the proposed approach we have considered two test cases. In both cases the result was positive, and the parameters were recovered with considerable precision even when the luminosity of the stars is poorly known. The consistency between the true and the inferred stellar parameters for both star 1 (computed with STAROX) and star 2 (computed with CESAM), seems to indicate that the method does not depend on the adopted evolutionary code. A comparison with other evolutionary codes (e.g. FRANEC, ATON, Siess et al. 2000) will be performed when a large number of accurate frequencies, as expected from CoRoT observations, will be available for PMS $\delta$ Scuti stars.

The method we propose is mainly aimed at studying stars whose pulsation data include several tens of frequencies, as it is expected to be obtained by CoRoT in the near future. However, we have also considered the possibility of applying it to stars whose frequency spectrum includes a smaller number of measured frequencies. To do so we have reported the preliminary applications to two stars that have been targets of multisite campaigns: V351 Ori and IP Per. The uniqueness of the solution is strongly dependent on the number of frequencies being used when these are less than about ten. However, the results illustrate the capability of this approach to constrain the stellar parameters, and in particular the stellar mass, even when a small number of frequencies is available.

For a higher number of frequencies the stellar mass and age can be constrained and the frequencies used to test the physics of the models. The application to a much larger number of frequencies for PMS pulsators (in particular as expected from space observations) and the adoption of finer model grids could considerably improve our knowledge of the stellar properties and structure in this very important phase of stellar evolution. A comparison with other evolutionary codes (e.g. FRANEC, ATON, Siess et al. 2000), as planned in the context of the CoRoT/ESTA collaboration, is also desirable.
Acknowledgements. We thank our referee W. Weiss for his valuable comments and suggestions. M.J.M. and J.P.M. were supported in part by FCT through project POCI/CTE-AST/57610/2004 from POCI, with funds from the European programme FEDER.

\section{References}

Alexander, D. R., \& Fergusson, J. W. 1994, ApJ, 437, 879

Baglin, A., and the CoRoT Team 2003, Adv. Space Res., 31, 345

Balona, L. A., Koen, C., \& van Wyk, F. 2002, MNRAS, 333, 923

Bernabei, S., Ripepi, V., Marconi, et al. 2004, in A-Star Puzzle, eds J. Zverko, J. Žižňovský, S. J. Adelman, \& W. W. Weiss (Cambridge University Press), 812

Böhm-Vitense, E. 1958, ZAp, 54, 114

Breger, M. 1972, ApJ, 171, 539

Breger, M., \& Bischof, K. M. 2002, A\&A, 385, 537

Christensen-Dalsgaard, J. 1982, MNRAS, 199, 735

Christensen-Dalsgaard, J., \& Gough D. O. 1982, MNRAS, 198, 141

Christensen-Dalsgaard, J., \& Berthomieu, G. 1991, in Solar Interior and Atmosphere, ed. A. N. Cox, W. C. Livingston, \& M. S. Matthews (University of Arizona Press), 401

Christensen-Dalsgaard, J., \& Mullan, D. J. 1994, MNRAS, 270, 921

Donati, J.-F., Semel, M., Carter, B. D., Rees, D. E., \& Cameron, A. C. 1997, MNRAS, 291, 658

Dziembowski, W. A. 1977, Acta Astron., 27, 203

Dziembowski, W. A., \& Goode, P. R. 1992, ApJ, 394, 670

Gough, D. O. 1993, Astrophysical fluid dynamics, Les Houches Session XLVII, ed. J.-P. Zahn, \& J. Zinn-Justin (Amsterdam: Elsevier), 399

Gough, D. O., \& Thompson, M. J. 1990, MNRAS, 242, 25

Grigahcéne, A. Duprey, M.-A., Garrido, R., Gabriel, M., \& Scuflaire, R. 2006, CoAst, 147, 69

Iglesias, C. A., \& Rogers, F. J. 1996, ApJ, 464, 943

Kurtz, D. W., \& Marang, F. 1995, MNRAS, 276, 191

Marconi, M., \& Palla, F. 1998, ApJ, 507, L141

Marconi, M., Palla, F. 2004, in A-Star Puzzle, ed. J. Zverko, J. Žižňovský, S. J. Adelman, \& W. W. Weiss (Cambridge University Press), 69

Marconi, M., Ripepi, V., Bernabei, S., et al. 2001, A\&A, 372, 21

Marconi, M., Ripepi, V., Palla, F., \& Ruoppo, A. 2004, CoAst, 145, 61

Marques, J. P. 2006, Ph.D. Thesis, Faculdade de ciências da Universidade do Porto

Marques, J. P., Fernandes, J., \& Monteiro, M. J. P. F. G. 2004, A\&A, 422, 239

Monteiro, M. J. P. F. G. 1996, Ph.D. Thesis, Queen Mary College, University of London, UK

Monteiro M. J. P. F. G., Lebreton Y., Montalbán J., et al. 2006, in The CoRoT Mission, ed. M. Fridlund, A. Baglin, J. Lochard, \& L. Conroy, ESA Publications Division, ESA SP-1306, 363

Morel, P. 1997, A\&AS, 124, 597

Palla, F., \& Stahler, S. W. 1993, ApJ, 418, 414

Pinheiro, F. J. G., Folha, D. F. M., Marconi, M., et al. 2003, A\&A, 399, 271

Ripepi, V., Palla, F., Marconi, M., et al. 2002, A\&A, 391, 587

Ripepi, V., Marconi, M., Bernabei, et al. 2003, A\&A, 408, 1047

Ripepi, V., Bernabei, S., Marconi, M., et al. 2006a, A\&A, 449, 335

Ripepi, V., Marconi, M., Bernabei, S., et al. 2006b, Mem. S.A.It., 77, 317

Rogers, F. J., Swenson, F. J., \& Iglesias, C. A. 1996, ApJ, 456, 902

Roxburgh, I. W. 2005, CoRoT/ESTA Meeting 4

(http://www . astro.up.pt/corot/welcome/meetings/m4/)

Soufi, F., Goupil, M. J., \& Dziembowski, W. A. 1998, A\&A, 334, 911

Siess, L., Dufour, E., \& Forestini, M. 2000, A\&A, 358, 593

Suran, M., Goupil, M., Baglin, A., Lebreton, Y., \& Catala, C. 2001, A\&A, 372, 233

Tassoul, M. 1980, ApJS, 43, 469

Templeton, M., \& Basu, S. 2003, in Asteroseismology Across the HR Diagram, ed. M. J. Thompson, M. S. Cunha, \& M. J. P. F. G. Monteiro (Kluwer Academic Publishers), 565 (CDRom)

Unno, W., Osaki, Y., Ando, H., \& Shibahashi, H. 1979, Nonradial Oscillations of Stars, (University of Tokyo Press)

Zwintz K., Marconi M., Kallinger T., \& Weiss W. W. 2004, in The A-Star Puzzle, ed. J. Zverko, J. Žižňovský, S. J. Adelman, \& W. W. Weiss (Cambridge University Press), IAU Symp., 224, 353

Matthews, J. 2007, CoAst, in press 